\title{
Thin-Film Quantum Dot Photodiode for Monolithic Infrared Image Sensors ${ }^{+}$
}

\author{
Pawel E. Malinowski ${ }^{1, *}$ (D) , Epimitheas Georgitzikis ${ }^{1,2}$, Jorick Maes ${ }^{3,4}$ (D) , Ioanna Vamvaka ${ }^{1,2}$, \\ Fortunato Frazzica ${ }^{1,5}$, Jan Van Olmen ${ }^{1}$, Piet De Moor ${ }^{1}$, Paul Heremans ${ }^{1,2}$, Zeger Hens ${ }^{3,4}$ and \\ David Cheyns ${ }^{1}$ \\ 1 IMEC, Kapeldreef 75, B-3001 Leuven, Belgium; Epimitheas.Georgitzikis@imec.be (E.G.); \\ Ioanna.Vamvaka.ext@imec.be (I.V.); Fortunato.Frazzica@imec.be (F.F.); Jan.VanOlmen@imec.be (J.V.O.); \\ Piet.DeMoor@imec.be (P.D.M.); Paul.Heremans@imec.be (P.H.); David.Cheyns@imec.be (D.C.) \\ 2 KU Leuven, Kasteelpark Arenberg 10, B-3001 Leuven, Belgium \\ 3 Physics and Chemistry of Nanostructures, Ghent University, Krijgslaan 281-S3, B-9000 Ghent, Belgium; \\ Jorick.Maes@UGent.be (J.M.); Zeger.Hens@UGent.be (Z.H.) \\ 4 Center for Nano- and Biophotonics (NB-Photonics), Ghent University, B-9000 Ghent, Belgium \\ 5 Vrije Universiteit Brussel (VUB-ETRO), Pleinlaan 2, B-1050 Brussel, Belgium \\ * Correspondence: Pawel.Malinowski@imec.be; Tel.: +32-1628-1938 \\ + This paper is an extended version of "Pawel E. Malinowski, Epimitheas Georgitzikis, Jorick Maes, \\ Mehedi Mamun, Oscar Enzing, Fortunato Frazzica, Jan Van Olmen, Piet De Moor, Paul Heremans, \\ Zeger Hens, and David Cheyns. Monolithic Near Infrared Image Sensors Enabled by Quantum Dot \\ Photodetector. In Proceedings of the 2017 International Image Sensor Workshop, Hiroshima, Japan, \\ 30 May-2 June 2017".
}

Received: 31 October 2017; Accepted: 8 December 2017; Published: 10 December 2017

\begin{abstract}
Imaging in the infrared wavelength range has been fundamental in scientific, military and surveillance applications. Currently, it is a crucial enabler of new industries such as autonomous mobility (for obstacle detection), augmented reality (for eye tracking) and biometrics. Ubiquitous deployment of infrared cameras (on a scale similar to visible cameras) is however prevented by high manufacturing cost and low resolution related to the need of using image sensors based on flip-chip hybridization. One way to enable monolithic integration is by replacing expensive, small-scale III-V-based detector chips with narrow bandgap thin-films compatible with 8- and 12-inch full-wafer processing. This work describes a CMOS-compatible pixel stack based on lead sulfide quantum dots (PbS QD) with tunable absorption peak. Photodiode with a 150-nm thick absorber in an inverted architecture shows dark current of $10^{-6} \mathrm{~A} / \mathrm{cm}^{2}$ at $-2 \mathrm{~V}$ reverse bias and EQE above 20\% at $1440 \mathrm{~nm}$ wavelength. Optical modeling for top illumination architecture can improve the contact transparency to $70 \%$. Additional cooling $(193 \mathrm{~K})$ can improve the sensitivity to $60 \mathrm{~dB}$. This stack can be integrated on a CMOS ROIC, enabling order-of-magnitude cost reduction for infrared sensors.
\end{abstract}

Keywords: infrared; imaging; image sensor; quantum dot; PbS; monolithic integration

\section{Introduction}

Near infrared (NIR) wavelength range $(0.7-1.4 \mu \mathrm{m})$ provides vital information in fields such as low-light/night vision, surveillance, sorting or biometrics, with content interpretation very similar to visible photography and imaging possible with no additional light source. Unfortunately, the sharply decreasing absorption of silicon around the wavelength of $900 \mathrm{~nm}$ (for standard photodiode thickness) prevents further extension of usable quantum efficiency range. At the same time, dedicated infrared sensors are not yet easily accessible due to their significantly higher cost than image sensors based on complementary metal-oxide-semiconductor (CMOS) technology, operating in the visible range. 
Typically, III-V semiconductor layers are used because of their sufficiently low energy bandgap [1,2]. Since they need to be grown by high-temperature epitaxy (and that on wafer sizes only up to 3-4 inch), already the starting material has orders-of-magnitude higher cost than standard silicon wafers. Moreover, flip-chip hybridization (usually die-to-die) is required which further increases the cost [3]. The ideal, simple solution would be to monolithically integrate an absorber layer directly on top of the silicon-based readout integrated circuit (ROIC, Figure 1).

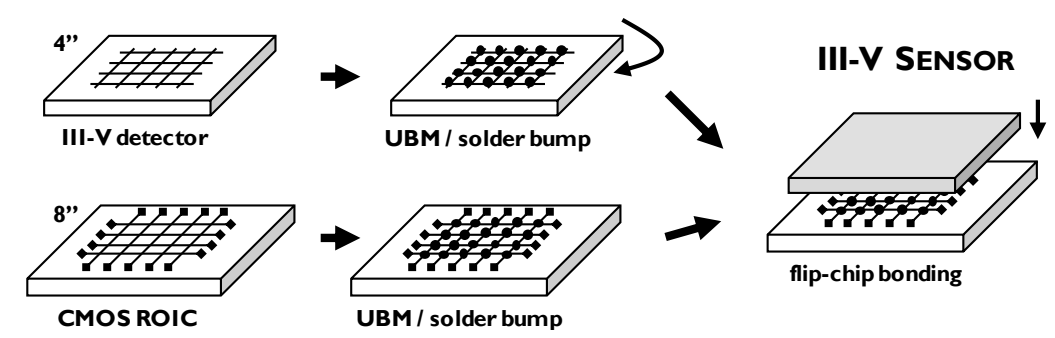

(a)

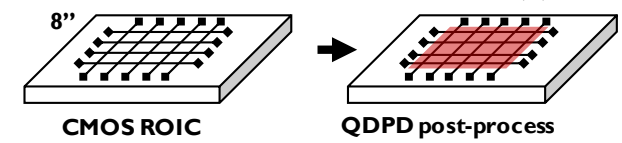

\section{QDPD SENSOR}

(b)

Figure 1. Integration route for a hybrid III-V infrared image sensor (a) and a monolithic quantum dot photodiode (QDPD) infrared image sensor (b).

Another aspect is the achievable resolution and pixel pitch of infrared image sensors. The traditional hybrid systems are typically limited to small arrays ( 1 megapixel range) due to small detector wafer size and low throughput. Pixel pitch does not go below $10 \mu \mathrm{m}$ [4] which is limited by the hybridization process-the solder bumps need sufficient volume for reliable bonding which in turn is limited by the achievable aspect ratio and pixel spacing. With a thin-film active layer integrated monolithically directly on top of the readout circuit, submicron pixel sizes $(0.9 \mu \mathrm{m}$ state-of-the-art for CMOS image sensors [5]) can be achieved (Figure 2).

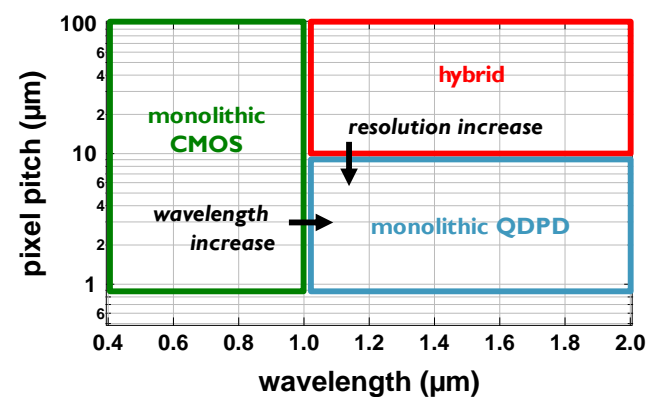

Figure 2. Positioning of a QDPD-based image sensor: higher wavelength than monolithic Si and higher resolution than hybrid alternatives.

Proof-of-concept image sensors with thin-film photodetector active layer have been demonstrated with different materials. Organic absorber integration has already been shown since more than 10 years ago on glass [6,7], flexible plastic foil $[8,9]$ and silicon readout [10]. There have been several demonstrations of organic thin-film photodetector integration on thin-film transistor (TFT) readout circuits, mostly for large area image sensors for X-ray radiography applications [11]. Recently, also perovskite absorbers were successfully integrated [12]. Organic films integration on CMOS ROIC followed a few improvement rounds by the same group, with the latest generation implemented with $0.9 \mu \mathrm{m}$ pixel size showing the potential of resolution scaling $[5,13,14]$. Near infrared imaging with solution processed thin-film photodetectors was shown with polymers [15] and quantum dots 
(QD) [16]. In this work, we describe building blocks for realization of a monolithic image sensor targeted for the infrared range up to the wavelengths close to $2 \mu \mathrm{m}$. Quantum Dot Photodetector (QDPD) is the device architecture of choice, with narrow-band PbS colloidal quantum dots [17-19] forming the absorber layer. These sensors can be fabricated without flip-chip hybridization of a III-V chip [20].

\section{Materials and Methods}

$\mathrm{PbS}$ quantum dots enable uncooled NIR detection up to $2 \mu \mathrm{m}$ wavelength, with the absorption peak tunable depending on the nanocrystal size (Figure 3). In this work, we describe two types of quantum dots: Larger (5.5 nm diameter), with the absorption peak at the wavelength of $1440 \mathrm{~nm}$ and smaller ( $3.4 \mathrm{~nm}$ diameter), with the peak at $980 \mathrm{~nm}$. The peak can be adjusted according to device specifications, for example with smaller dots to add near infrared bands to hyperspectral visible image sensors and with larger dots to address the spectrum of InGaAs image sensors.

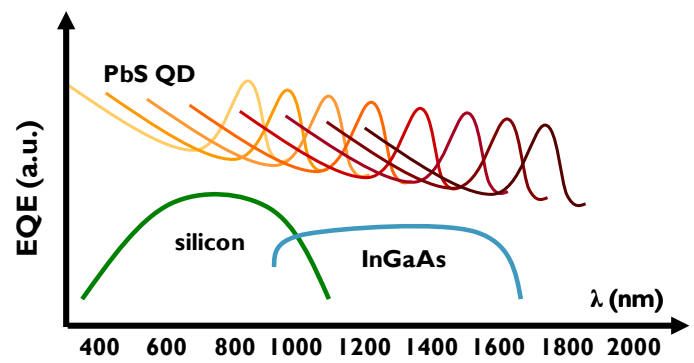

(a)

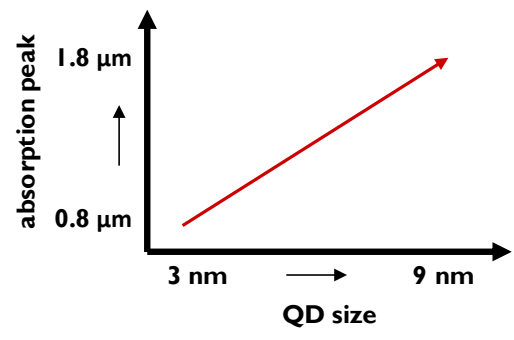

(b)

Figure 3. Schematic external quantum efficiency curves for silicon, InGaAs and PbS QD photodetectors (a) and indicative absorption peak dependence on quantum dot size (b).

In our investigation, we take a stepwise approach to develop a photodetector stack that can be used on top of a readout integrated circuit (ROIC) based on complementary metal-oxide-semiconductor (CMOS) technology (Figure 4). As the first step, we use glass substrates to test the coating feasibility of the colloidal quantum dot solution. Such structure enables optimization of film parameters such as thickness, morphology and uniformity, as well as elaboration of the absorption profile. As the next step, glass substrates with pre-patterned indium tin oxide (ITO) contacts are used to fabricate a photodetector stack with all additional layers, such as electron transport layer (ETL), hole transport layer (HTL) and injection/blocking layers. This stack can be characterized electrically and under bottom illumination (through the substrate). Once the photodiode performance is established, the stack is transferred to $\mathrm{Si} / \mathrm{SiO}_{2}$ substrates with metal bottom contact to imitate the CMOS ROIC architecture. TiN is used as the contact as it is one of the standard materials in the CMOS flow [21]. Here, we adjust the stack to operate in top illumination condition, which includes changing the top contact to a stack that is as transparent as possible in the wavelength range of interest and tuning the thicknesses of all layers to harness the maximum amount of incoming light [22].

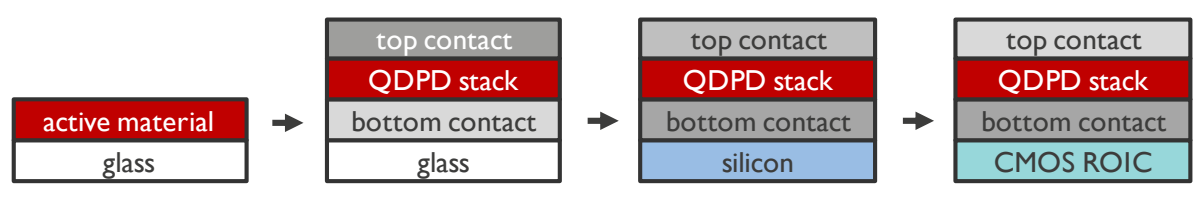

Figure 4. Methodology used for the development of CMOS-compatible QDPD pixel stacks.

The fabrication process starts by cleaning the substrates with a standard detergent-watersolvent procedure. A metal-oxide electron transport layer (ETL) is then deposited to form an inverted architecture of the photodiode (with photo-generated electrons collected at the readout chip side). 
Examples of this layer are $\mathrm{TiO}_{x}$ or $\mathrm{ZnO}$ (n-type semiconductors) that improve electron transport and injection. The quantum dots are deposited by spin-coating from a colloidal solution as a multilayer stack to form a $150 \mathrm{~nm}$ thick active layer. The total thickness can be adjusted by the number of sublayers and by the thickness of each sublayer. On top of the stack, an organic ( $p$-type polymer) hole transport layer (HTL) is deposited to improve hole transport and injection, followed by a top contact (either thick, opaque metal for bottom illumination or semi-transparent metal for top illumination).

\section{Results}

\subsection{Quantum Dot Film}

Test substrates with a spin-coated three-sublayer stack of the quantum dot film are characterized with Transmission Electron Microscope (TEM). The inspection reveals perfect crystallinity within each nanocrystal, high level of crystallinity within a single sublayer and random orientation between sublayers (Figure 5). In the high magnification image, one can also see a uniform size distribution of the QDs.

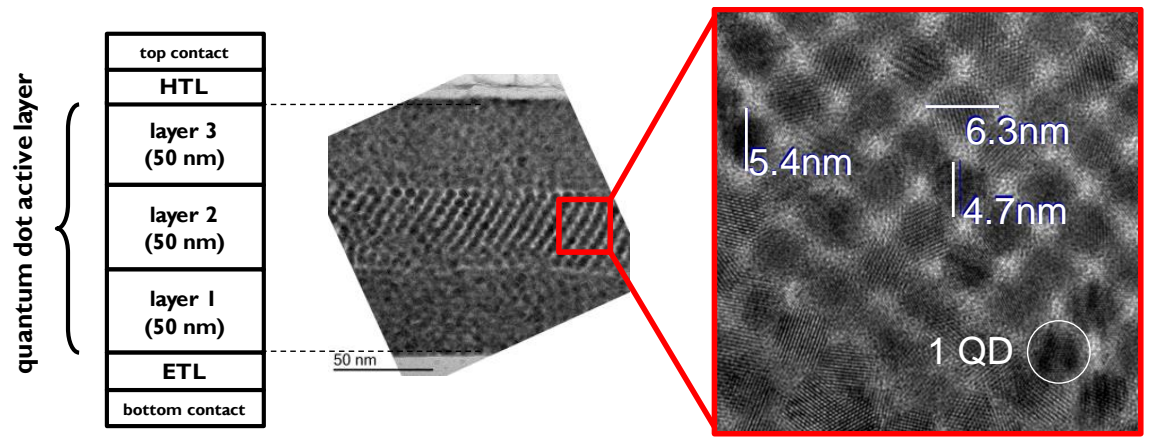

Figure 5. Cross-section (left: schematic; right: Transmission Electron Micrograph) of a 3-layer active stack based on $5.5 \mathrm{~nm}$ PbS quantum dots.

\subsection{QDPD on Glass}

Full photodetector stacks including contacts and transport layers are fabricated on glass/ITO test vehicles. Each $3 \times 3 \mathrm{~cm}^{2}$ glass substrate has 12 single pixel devices, each with an active area of $0.125 \mathrm{~cm}^{2}$. Current density-voltage characterization (Figure 6a) of a baseline stack using $5.5 \mathrm{~nm}$ QDs shows dark current density of $1 \mu \mathrm{A} / \mathrm{cm}^{2}$ at $-2 \mathrm{~V}$ reverse bias voltage. The external quantum efficiency (EQE, Figure 6b) shows that by choosing the size of the quantum dot in the active layer, the absorption peak can be tuned in a wide wavelength range (here, $1020 \mathrm{~nm}, 1250 \mathrm{~nm}$ and $1440 \mathrm{~nm}$ ). The full width at half maximum (FWHM) for the EQE is approximately $100 \mathrm{~nm}$.

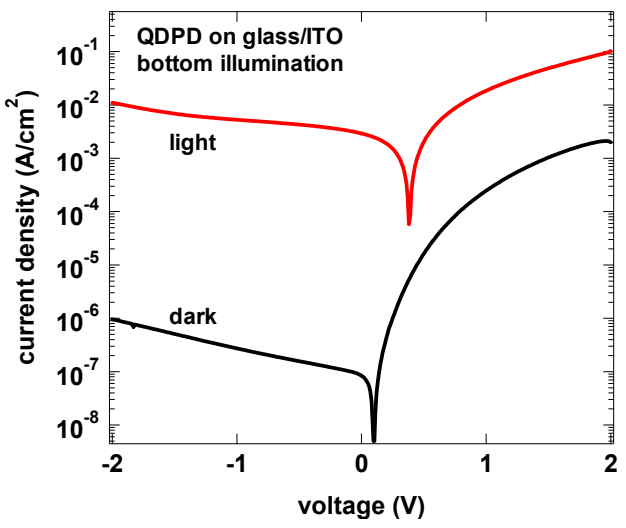

(a)

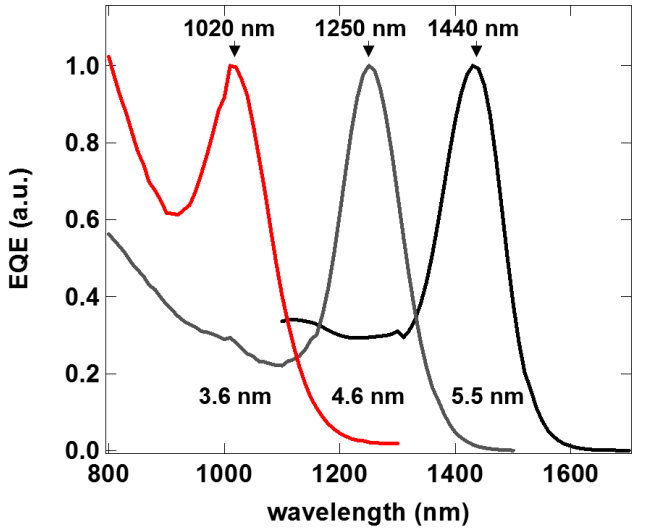

(b)

Figure 6. Current density vs. voltage of a bottom illuminated QDPD (a) and external quantum efficiency curves for different QD sizes used in the photoactive layer (b). 
External quantum efficiency of the quantum dot photodetectors extends over a wide range of wavelengths (above $300 \mathrm{~nm}$ ) and then features a characteristic absorption peak related to the quantum dot size. In the bottom illumination architecture, we obtain 11\% EQE at the wavelength of $980 \mathrm{~nm}$ for QDs with a diameter of $3.4 \mathrm{~nm}$, with above $60 \%$ EQE between $350 \mathrm{~nm}$ and $500 \mathrm{~nm}$ (Figure 7a). For the larger QDs of $5.5 \mathrm{~nm}$, the peak lies at $1440 \mathrm{~nm}$, with EQE of 22\% (Figure 7b). Taking the photodiode dark current as the major noise component, we can obtain specific detectivity $\left(D^{*}\right)$ of $3 \times 10^{11}$ Jones.

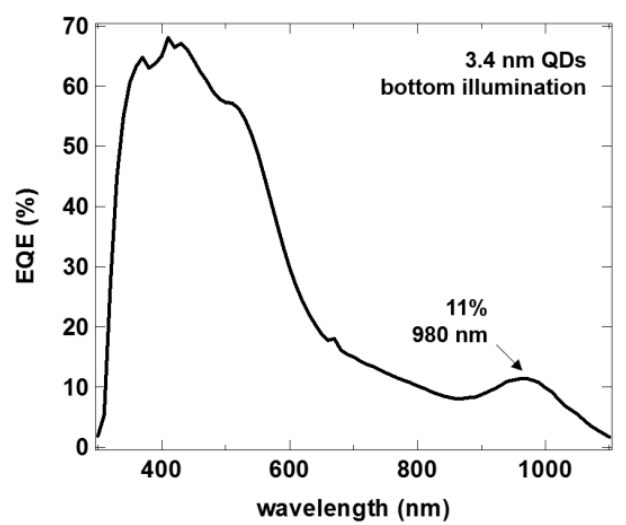

(a)

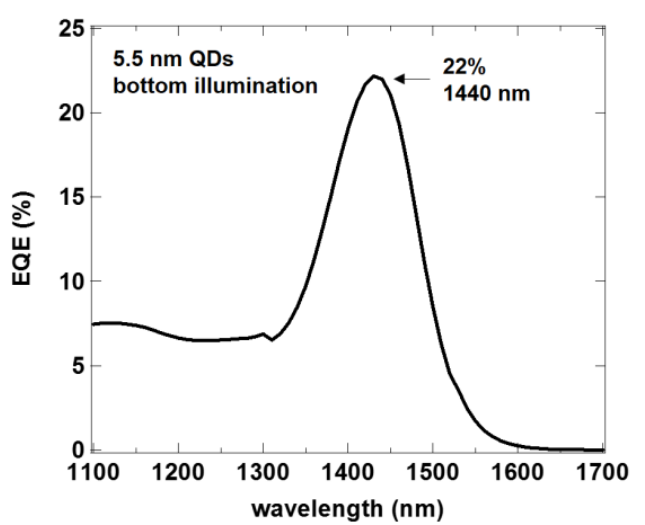

(b)

Figure 7. External quantum efficiency of QDPD devices with $3.4 \mathrm{~nm}$ (a) and $5.5 \mathrm{~nm}$ (b) quantum dots.

\subsection{QDPD on Silicon}

After the first optimization of the stack on glass/ITO, the stacks are transferred to $3 \times 3 \mathrm{~cm}^{2}$ silicon substrates with TiN bottom contact. The contact is structured so that different active areas are available to investigate scaling effects of the dark current. Each substrate has many test vehicles with pixel sizes of $2 \times 2 \mathrm{~mm}^{2}$ down to $50 \times 50 \mu^{2}$. An inorganic edge cover layer (ECL) is used to precisely define the active area and exclude the effects of the fanout structure (Figure 8).

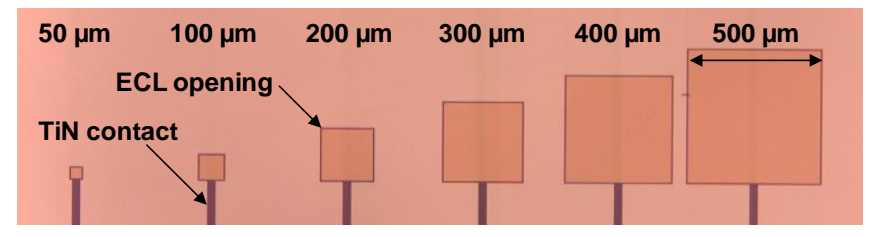

Figure 8. Microscope image of the silicon substrate with different pixel sizes showing TiN bottom contact and inorganic edge cover layer.

Dark current density on silicon/TiN substrates equals $6 \times 10^{-3} \mathrm{~mA} / \mathrm{cm}^{2}$ at $-2 \mathrm{~V}$ which shows that the photodetector can be fabricated on the CMOS-compatible bottom contact (Figure 9a). Each pixel size showed also the same current density, indicating linear scaling of the dark current with active area (Figure 9b). Photocurrent was measured with an infrared light emitting diode $\left(5 \mathrm{~mW} / \mathrm{cm}^{2}\right.$ LED power, $1450 \mathrm{~nm}$ center wavelength) in top illumination (through the semi-transparent top contact) and followed the same trend. 


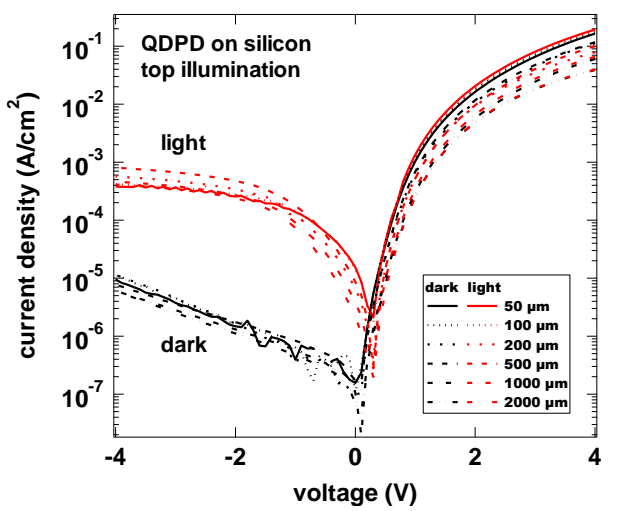

(a)

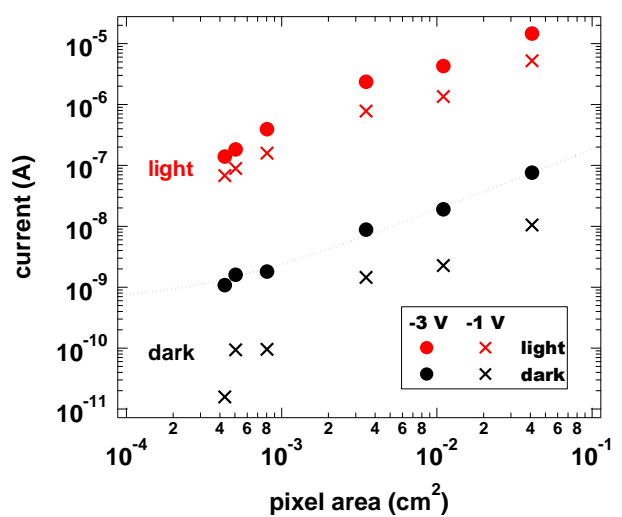

(b)

Figure 9. Dark and photocurrent density vs. voltage curves (a); and current vs. pixel area curves (b) for active areas between $50 \times 50 \mu \mathrm{m}^{2}$ and $2 \times 2 \mathrm{~mm}^{2}$.

In top illumination tests with IR LED and increasing irradiance, we could observe a linear increase of the photocurrent (Figure 10). The calculated photo-to-dark current ratio is between 32 and $43 \mathrm{~dB}$ in the reverse bias voltage range of -3 to $-5 \mathrm{~V}$, respectively.

Indium tin oxide (ITO) bottom contact used in the bottom illuminated test vehicle is not ideal for the top illuminated silicon substrates as it has a limited transparency in the infrared wavelength range ( $80 \%$ at $1100 \mathrm{~nm}$ and $55 \%$ at $1440 \mathrm{~nm}$ ). To optimize the semi-transparent top contact for absorption in the NIR range, we used optical interference modelling with transfer matrix method. We obtained transparency of $70 \%$ (Figure 11) which was verified experimentally. This shows a significant boost from the standard contact structure used in the visible range. In this way, the EQE of top-illuminated photodetectors on TiN bottom contact can be further improved and reach $25 \%$ at the wavelength of $1440 \mathrm{~nm}$, even though the active layer thickness is only $150 \mathrm{~nm}$.

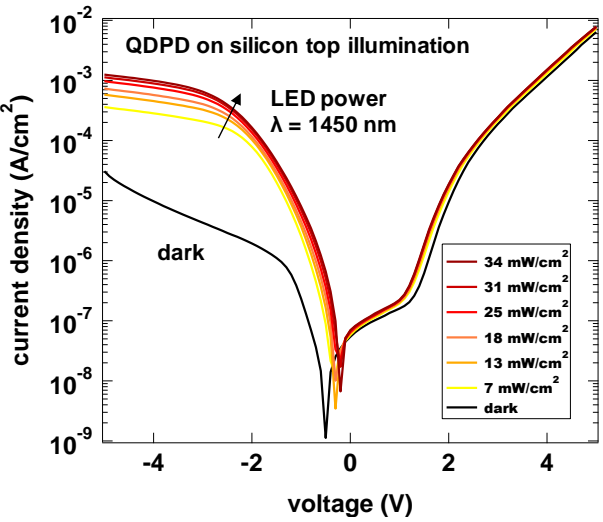

(a)

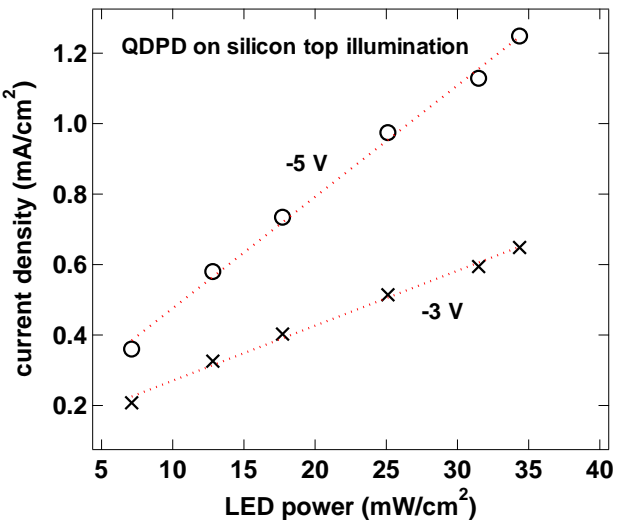

(b)

Figure 10. Current density—voltage characteristics (a) of a QDPD on Si substrate in dark (black line) and under $1450 \mathrm{~nm}$ IR LED with varying power (color lines); and linearity curve of the photocurrent density (b). 


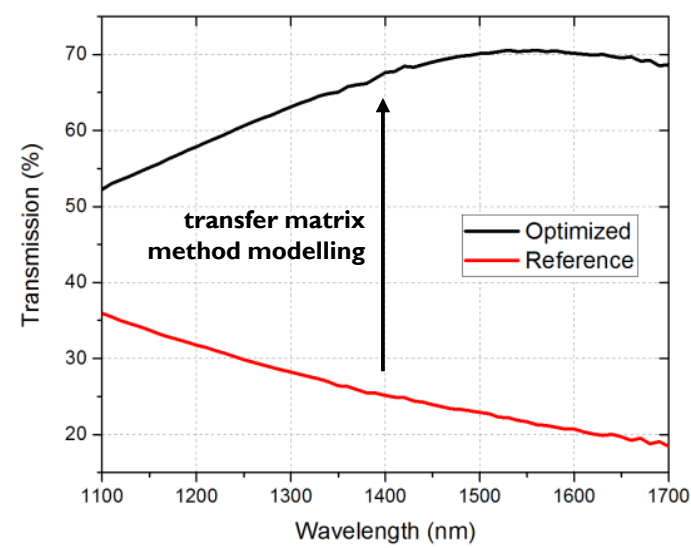

(a)

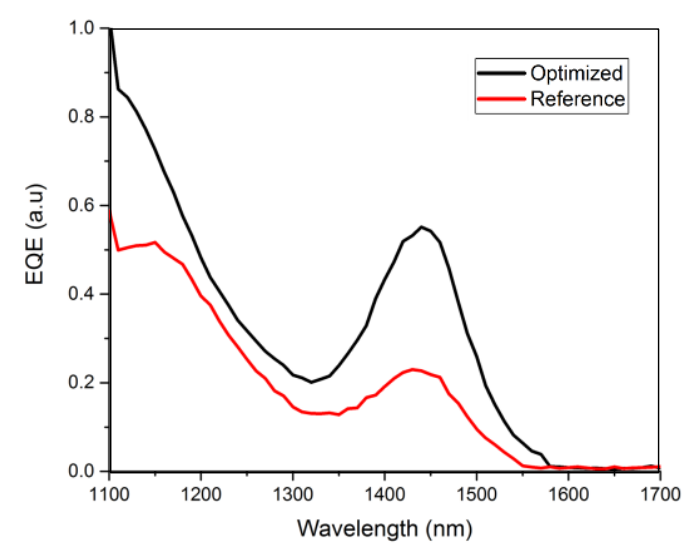

(b)

Figure 11. Top illuminated photodetector stack optimized for NIR transparency: Improvement of the top contact transparency (a); and experimental EQE verification (b).

\subsection{QDPD Additional Characterization}

Even though PbS QDPDs enable detection at room temperature, standard IR camera packaging offers possibility of additional cooling. In the cryostat measurements, we observed that by cooling the detector to $193 \mathrm{~K}$ we can further improve the current ratio to over $60 \mathrm{~dB}$ from $30 \mathrm{~dB}$ at room temperature (Figure 12). Depending on the application, this might be a way to boost the sensitivity of the image sensor.

Speed of the QDPDs was measured using an oscilloscope for a photodiode with an area of $0.041 \mathrm{~cm}^{2}$. While keeping the reverse bias voltage at $-2 \mathrm{~V}$, the infrared LED light source $(1450 \mathrm{~nm}$ center wavelength) was switched on and off. Rise time of $13 \mu$ s and fall time of $41 \mu$ s were measured (Figure 13). Such performance is sufficient for basic imaging, but a further speed improvement will enable other applications.

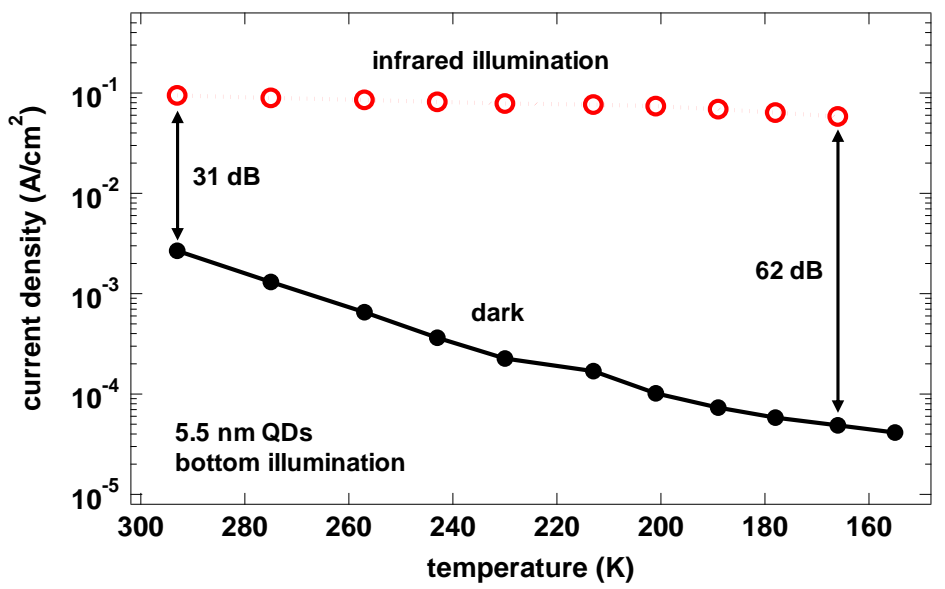

Figure 12. Dark and photocurrent vs. photodetector temperature, indicating an increasing current ratio with lowering the operating temperature. 


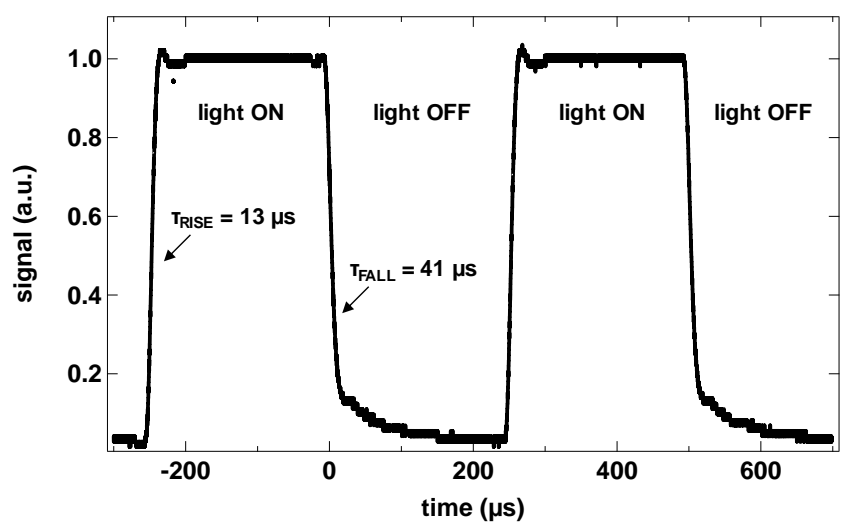

Figure 13. Transient characteristics of a test QDPD device with an active area of $0.041 \mathrm{~cm}^{2}$ and under illumination with a switching NIR LED.

\section{Discussion}

$\mathrm{PbS}$ quantum dots are implemented in photodetector stacks targeting detection of near infrared radiation. Dark current density is in the range of $10^{-6} \mathrm{~A} / \mathrm{cm}^{2}$ at $-2 \mathrm{~V}$ reverse bias voltage. This value is several orders of magnitude higher than benchmark silicon-based photodetectors $\left(10^{-12} \mathrm{~A} / \mathrm{cm}^{2}-10^{-10} \mathrm{~A} / \mathrm{cm}^{2}\right)$, and within an order of magnitude as compared to InGaAs-based infrared photodetectors operating at similar conditions [2]. Other quantum dot photodetectors show different values of dark current density $\left(10^{-8} \mathrm{~A} / \mathrm{cm}^{2}-10^{-3} \mathrm{~A} / \mathrm{cm}^{2}\right)$, depending on the QD size, ligands, transport layers used in the stack and characterization conditions [16,23,24]. Even though it is difficult to have a direct comparison between published results, the photodetectors presented here have comparable dark current, especially taking into account the stack design for higher cut-off wavelength (thus with a lower energy bandgap). Here, the dark current values are given for the $5.5 \mathrm{~nm}$ QDs $(1440 \mathrm{~nm})$ and they are expected to be lower for the smaller diameters (e.g., for $940 \mathrm{~nm}$ ). At the same time, the best organic photodetectors with similar thin-film multilayer stacks feature dark currents comparable to silicon $\left(10^{-11} \mathrm{~A} / \mathrm{cm}^{2}\right.$ after [11]), indicating that the leakage current might not be limited by the thin-film stack but rather by the narrow-bandgap semiconductor and its interfaces. Current density scales linearly for pixel sizes between $2 \times 2 \mathrm{~mm}^{2}$ and $50 \times 50 \mu^{2}$, indicating no perimeter effects down to this active area. For smaller pixels, active readout is necessary due to the very low current levels.

External quantum efficiency (EQE) is above $20 \%$ at the absorption peak at the wavelength of $1440 \mathrm{~nm}$ in a 150-nm thick active layer. Even though this is still significantly lower than EQE of InGaAs photodetectors, it is at the same time unachievable by Si photodetectors. Moreover, we have also demonstrated stacks with other absorption peaks, as they can be quite accurately tuned by the QD diameter. For a $980-\mathrm{nm}$ peak stack, the EQE is above $10 \%$ and is currently under optimization, expected to exceed $40-50 \%$. As the QDPD can be integrated on top of the CMOS ROIC, one might imagine not only monochromatic infrared imagers, but also extension of current hyperspectral visible imagers in combination with a standard silicon pinned photodiode.

As the QDPD stack will be integrated on top of a CMOS ROIC, the pixel stack is optimized for operation in a top illuminated architecture with a TiN bottom contact. We have observed similar electrical performance as in reference devices on glass substrates with ITO contact. Photocurrent and thus efficiency was improved by tuning the thicknesses of all layers in the stack with optical simulations. The transfer matrix method can be used to maximize performance of thin films for the wavelength of interest. We have calculated that the EQE achievable with $\mathrm{PbS}$ is about $30 \%$ for the wavelength of $1440 \mathrm{~nm}$.

Even though one of the advantages of the PbS photodetector is operability at room temperature, we have seen that by cooling the device to $193 \mathrm{~K}$, the photo-to-dark current ratio can be increased from $30 \mathrm{~dB}$ to $60 \mathrm{~dB}$. This shows the potential of higher sensitivity for specific applications. 
The photodetector speed is sufficient for imaging, but is still a parameter of concern. Currently, we are investigating possible limiting factors (e.g., trap states, interface defects) and optimizing the charge transfer properties of the stack to reach switching speeds in the range of tens of ns.

The next step of development is integration of the photodetector stack on the CMOS ROIC test chip. Research activities include lifetime studies (to verify the encapsulation specifications), photolithographic patterning feasibility (to enable side-by-side multicolor arrays) and packaging aspects. The monolithic QDPD integration method will enable low-cost infrared cameras with resolution and pitch limited only by the ROIC design.

\section{Conclusions}

Stacks using $\mathrm{PbS}$ quantum dot absorber layer can be used as efficient near infrared photodetectors even though the total thickness is only in the range of $100 \mathrm{~nm}$. This makes them an interesting candidate for integration on top of CMOS readout circuits, thus enabling monolithic image sensors that have an order-of-magnitude lower cost figure than hybrid devices and are not limited by the resolution and array size of flip-chip hybridization.

The quantum dot photodetector stack can be fabricated using standard semiconductor processing methods in the fab environment. 8- or 12-inch substrates can be used. In the final camera system, the detailed optical design will strongly depend on the wavelength of interest for the application. For the case of extension of visible CMOS image sensor to NIR, the standard optics found in commercial cameras might be used, while infrared optics will be necessary for a monochrome NIR imager for the higher wavelengths towards $2 \mu \mathrm{m}$.

In summary, the pixel stack demonstrated here shows building blocks for fabrication of a monolithic image sensor for the near infrared wavelength range. The benefits of using QDPD active stack are ease of processing, room-temperature operation, submicron active layer thickness and high EQE in NIR.

Acknowledgments: The results reported in this paper have been realized with the funding of Agentschap Innoveren \& Ondernemen (VLAIO) in the framework of project MIRIS (IWT/150029).

Author Contributions: P.E. Malinowski coordinated the project and wrote the paper, E. Georgitzikis devised and performed the experiments, characterization and data analysis, J. Maes devised and synthesized the quantum dot material, I. Vamvaka fabricated and characterized test devices, F. Frazzica designed the readout architecture, J. Van Olmen fabricated the test structures, P. De Moor, P. Heremans and Z. Hens supervised the research teams, and D. Cheyns devised and supervised the scientific activity.

Conflicts of Interest: The authors declare no conflict of interest.

\section{References}

1. Martyniuk, P.; Antoszewski, J.; Martyniuk, M.; Faraone, L.; Rogalski, A. New concepts in infrared photodetector designs. Appl. Phys. Rev. 2014, 041102. [CrossRef]

2. Rogalski, A. Progress in focal plane array technologies. Prog. Quantum Electron. 2012, 36, 342-473. [CrossRef]

3. John, J.; Zimmermann, L.; Nemeth, S.; Colin, T.; Merken, P.; Borghs, S.; Van Hoof, C.A. Extended InGaAs on GaAs Detectors for SWIR Linear Sensors. Proc. SPIE 2001, 4369, 692-697. [CrossRef]

4. Malinowski, P.E.; Duboz, J.-Y.; De Moor, P.; John, J.; Minoglou, K.; Srivastava, P.; Semond, F.; Frayssinet, E.; Giordanengo, B.; BenMoussa, A.; et al. AlGaN-on-Si-Based 10- $\mu \mathrm{m}$ Pixel-to-Pixel Pitch Hybrid Imagers for the EUV Range. IEEE Electron Device Lett. 2011, 32, 1561-1563. [CrossRef]

5. Takase, M.; Miyake, Y.; Yamada, T.; Tamaki, T.; Murakami, M.; Inoue, Y. First Demonstration of $0.9 \mu \mathrm{m}$ Pixel Global Shutter Operation by Novel Charge Control in Organic Photoconductive Film. In Proceedings of the 2015 IEEE International Electron Devices Meeting (IEDM), Washington, DC, USA, 7-9 December 2015. [CrossRef]

6. Yu, G.; Wang, J.; McElvain, J.; Heeger, A.J. Large-Area, Full-Color Image Sensors Made with Semiconducting Polymers. Adv. Mater. 1998, 10, 1431. [CrossRef]

7. Tedde, S.; Zaus, E.S.; Fürst, J.; Henseler, D.; Lugli, P. Active Pixel Concept Combined with Organic Photodiode for Imaging Devices. IEEE Electron Device Lett. 2007, 28, 893-895. [CrossRef] 
8. Someya, T.; Kato, Y.; Iba, S.; Noguchi, Y.; Sekitani, T.; Kawaguchi, H.; Sakurai, T. Integration of Organic FETs With Organic Photodiodes for a Large Area, Flexible, and Lightweight Sheet Image Scanners. IEEE Trans. Electron Devices 2005, 52, 2502-2511. [CrossRef]

9. Malinowski, P.E.; Kumar, A.; Moet, D.J.D.; Cheyns, D.; Rand, B.P.; van der Steen, J.-L.P.J.; Myny, K.; Steudel, S.; Simon, M.; Gelinck, G.; et al. Fully Organic Integrated Arrays on Flexible Substrates for X-ray Imaging. In Proceedings of the 2013 International Image Sensor Workshop, Snowbird, UT, USA, 12-16 June 2013; Volume 47.

10. Takada, S.; Ihama, M.; Inuiya, M. CMOS image sensor with organic photoconductive layer having narrow absorption band and proposal of stack type solid-state image sensors. Proc. SPIE 2006, 6068, 6068-6612. [CrossRef]

11. Gelinck, G.H.; Kumar, A.; Moet, D.; van der Steen, J.-L.P.J.; van Breemen, A.J.J.M.; Shanmugam, S.; Langen, A.; Gilot, J.; Groen, P.; Andriessen, R.; et al. X-ray Detector-on-Plastic with High Sensitivity Using Low Cost, Solution-Processed Organic Photodiodes. IEEE Trans. Electron Devices 2016, 63. [CrossRef]

12. Kim, Y.C.; Kim, K.H.; Son, D.-Y.; Jeong, D.-N.; Seo, J.-Y.; Choi, Y.S.; Han, I.T.; Lee, S.Y.; Park, N.-G. Printable organometallic perovskite enables large-area, low-dose X-ray imaging. Nature 2017, 550, 87. [CrossRef] [PubMed]

13. Ihama, M.; Inomata, H.; Asano, H.; Imai, S.; Mitsui, T.; Imada, Y.; Hayashi, M.; Gotou, T.; Suzuki, H.; Sawaki, D.; et al. CMOS Image Sensor with an Overlaid Organic Photoelectric Conversion Layer. In Proceedings of the 2011 International Image Sensor Workshop, Hokkaido, Japan, 8-11 June 2011.

14. Ihama, M.; Koguchi, H.; Inomata, H.; Asano, H.; Imada, Y.; Mishima, Y.; Kato, Y.; Hirose, Y.; Segawa, M.; Kishimura, T.U.; et al. Organic CMOS Image Sensor with Thin Panchromatic Organic Photoelectric Conversion Layer. In Proceedings of the 2013 International Image Sensor Workshop, Snowbird, UT, USA, 12-16 June 2013.

15. Baierl, D.; Pancheri, L.; Schmidt, M.; Stoppa, D.; Betta, G.-F.D.; Scarpa, G.; Lugli, P. A hybrid CMOS-imager with a solution-processable polymer as photoactive layer. Nat. Commun. 2012, 3, 1175. [CrossRef] [PubMed]

16. Rauch, T.; Boberl, M.; Tedde, S.F.; Furst, J.; Kovalenko, M.V.; Hesser, G.N.; Lemmer, U.; Heiss, W.; Hayden, O. Near-infrared imaging with quantum-dot-sensitized organic photodiodes. Nat. Photonics 2009, 3, 332. [CrossRef]

17. Hu, C.; Gassenq, A.; Justo, Y.; Devloo-Casier, K.; Chen, H.; Detavernier, C.; Hens, Z.; Roelkens, G. Air-stable short-wave infrared $\mathrm{PbS}$ colloidal quantum dot photoconductors passivated with $\mathrm{Al}_{2} \mathrm{O}_{3}$ atomic layer deposition. Appl. Phys. Lett. 2014, 105, 171110. [CrossRef]

18. Clifford, J.P.; Konstantatos, G.; Johnston, K.W.; Hoogland, S.; Levina, L.; Sargent, E.H.F. Sensitive and spectrally tuneable colloidal quantum-dot photodetectors. Nat. Nanotechnol. 2009, 4, 40-44. [CrossRef] [PubMed]

19. Hendricks, M.P.; Campos, M.P.; Cleveland, G.T.; Jen-La Plante, I.; Owen, J.S. A tunable library of substituted thiourea precursors to metal sulfide nanocrystals. Science 2015, 348, 1226-1230. [CrossRef] [PubMed]

20. Malinowski, P.E.; Georgitzikis, E.; Maes, J.; Mamun, M.; Enzing, O.; Frazzica, F.; Van Olmen, J.; De Moor, P.; Heremans, P.; Hens, Z; et al. Monolithic Near Infrared Image Sensors Enabled by Quantum Dot Photodetector. In Proceedings of the 2017 International Image Sensor Workshop, Hiroshima, Japan, 30 May-2 June 2017; Volume R54.

21. Solovan, M.N.; Brusa, V.V.; Maistruk, E.V.; Maryanchuk, P.D. Electrical and Optical Properties of TiN Thin Films. Inorg. Mater. 2014, 50, 40-45. [CrossRef]

22. Georgitzikis, E.; Malinowski, P.E.; Mamun, M.; Enzing, O.; Maes, J.; Hens, Z.; Heremans, P.; Cheyns, D. Determining charge carrier extraction in lead sulfide quantum dot near infrared photodetectors. Proc. SPIE 2017, 10348, 103480B. [CrossRef]

23. Pal, B.N.; Robel, I.; Mohite, A.; Laocharoensuk, R.; Werder, D.J.; Klimov, V.I. High-Sensitivity p-n Junction Photodiodes Based on PbS Nanocrystal Quantum Dots. Adv. Funct. Mater. 2012, 22, 1741-1748. [CrossRef]

24. Azmi, R.; Aqoma, H.; Tantyo Hadmojo, W.; Yun, J.M.; Yoon, S.; Kim, K.; Rag Do, Y.; Oh, S.H.; Jang, S.Y. Low-Temperature-Processed 9\% Colloidal Quantum Dot Photovoltaic Devices through Interfacial Management of p-n Heterojunction. Adv. Energy Mater. 2016, 1502146. [CrossRef]

(C) 2017 by the authors. Licensee MDPI, Basel, Switzerland. This article is an open access article distributed under the terms and conditions of the Creative Commons Attribution (CC BY) license (http://creativecommons.org/licenses/by/4.0/). 